\title{
A Fast Translucency Appearance Model
}

\author{
Francesco Banterle* \\ University of Verona
}

\section{Introduction}

Translucency is an important feature for the representation of some real world materials such as marble, jade, human skin and etc. We present a new technique for rendering the appearance of translucent material in real-time based on an image based approach. This technique isn't physically based as the dipole approximation [Jensen et al. 2001], but it reaches high frame rates, so it could be used in interactive applications.

\section{Proposed Model}

The key idea of the technique is that, when light enters in an object from an incoming point $\left(x_{i n}\right)$, it is focused in a point near surface called accumulation point $\left(x_{a}\right)$. Then light is transmitted in the object according to its index of refraction, and it leaves object from a outgoing point $\left(x_{\text {out }}\right)$.

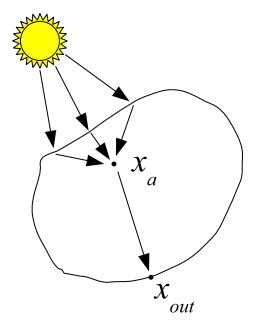

(a)

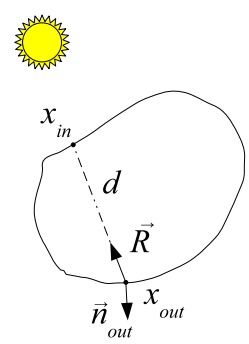

(b)
Figure 1: The appearance model: a) accumulation step, b) evaluation step.

We render the translucent object into a cube map (acquired from the faces of the objects bounding box, this constraints to convex object) with only direct lighting. So we project the radiance of cubemap into spherical harmonics $(\mathrm{SH})$ basis $L_{l m}$ using nine coefficients [Ramamoorthi and Hanrahan 2001]. With this step the light is accumulated in $x_{a}$ Fig.1(a).

In order to evaluate the irradiance at point $x_{\text {out }}$ we calculate refraction vector $\vec{R}$ of light vector $\vec{L}$ in $x_{\text {out }}, \vec{R}$ is used to evaluate $\mathrm{SH}$ and to get irradiance $E\left(x_{\text {out }}, \vec{n}_{\text {out }}\right)$. Finally this value is scaled to take account of density of material, by this term $d \sigma_{1} e^{-d \sigma_{2}}$. Where $\sigma_{1}$ is the scattering coefficient, $\sigma_{2}$ is the absorption coefficient, and $d$ is the distance from $x_{\text {in }}$ to $x_{\text {out }}$ calculated from the light's shadow map Fig.1(b).

\section{Results}

We implemented the proposed technique on GPU (projection on SH basis, and $\mathrm{SH}$ evaluation), in a real-time 3D application using DirectX9c and HLSL shading language. For all the results, we have used an Intel Pentium4 $2.8 \mathrm{GHz}$ PC, equipped with $1 \mathrm{~GB}$ RAM and an NVIDIA GeForce6800 graphics board. The size of a face of

\footnotetext{
*e-mail: frabante@gmail.com
}

cube map was $64 \times 64$ and the rendering viewport was $512 \times 512$. We achieve a frame rate of $67 \mathrm{fps}$ for the Happy Buddha model Fig.2, and $81 \mathrm{fps}$ for the Dragon Fig.3.
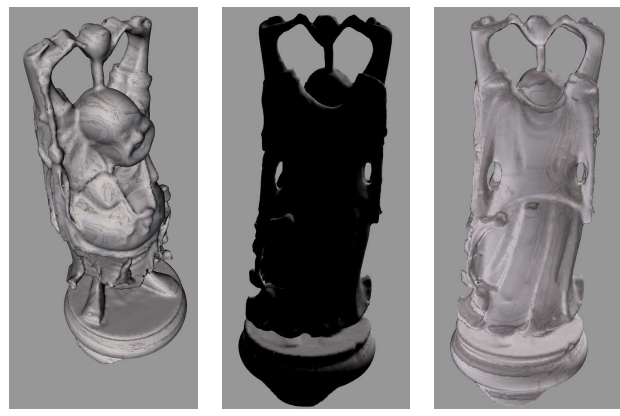

Figure 2: Happy Buddha ( $\sim 70 \mathrm{~K}$ triangles): Left direct lighting only from light view point. Middle direct lighting only behind the light. Right Rendering with direct lighting and subsurface scattering (67fps).
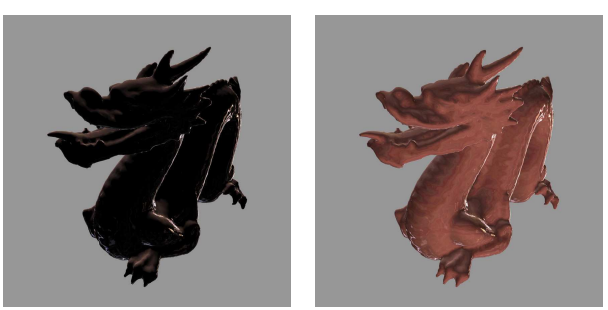

Figure 3: Stanford's Dragon ( $\sim 50 \mathrm{~K}$ triangles): Left direct lighting only behind the light. Right rendering with direct lighting and subsurface scattering (81fps).

\section{Conclusion}

We presented a fast, GPU friendly, translucency model suitable for convex objects (errors occur for concave object, but often visually unnoticeable). As the main projection step and SH evaluation can efficiently exploit the graphics hardware, this technique allows the interactive applications to represent translucent materials in real-time at a low cost and without any constraint in terms of light conditions and animations (deformable meshes are supported).

Acknowledgements: The 3D models are courtesy of the Stanford University Computer Graphics Lab.

\section{References}

Jensen, H. W., Marschner, S. R., Levoy, M., And Hanrahan, P. 2001. A practical model for subsurface light transport. In SIGGRAPH '01: Proceedings of the 28th annual conference on Computer graphics and interactive techniques, ACM Press, 511-518.

RAMAMOORTHI, R., AND HANRAHAN, P. 2001. An efficient representation for irradiance environment maps. In SIGGRAPH '01: Proceedings of the 28th annual conference on Computer graphics and interactive techniques, ACM Press, 497500. 